\title{
Energy-saving Optimal Scheduling of Laminar Cooling Water Supply Pumping Stations in Hot Rolling Mills Considering Complex Intermittent Working Conditions
}

\section{Fenjia Wang}

University of Science and Technology Beijing

Yong Song ( $\sim$ songyong@ustb.edu.cn )

University of Science and Technology Beijing https://orcid.org/0000-0003-2779-7075

Chao Liu

University of Science and Technology Beijing

Anrui He

University of Science and Technology Beijing

\section{Wei Zhao}

Anshan Iron and Steel Group Company Limited

\section{Research Article}

Keywords: Industrial energy saving, Laminar cooling, Intermittent production, On-line Sequential Extreme Learning Machine, Improved sparrow search algorithm

Posted Date: February 15th, 2022

DOI: https://doi.org/10.21203/rs.3.rs-1199733/v1

License: (c) (1) This work is licensed under a Creative Commons Attribution 4.0 International License.

Read Full License 


\title{
Energy-saving optimal scheduling of laminar cooling water supply pumping
}

\section{stations in hot rolling mills considering complex intermittent working conditions}

Fenjia Wang ${ }^{1} \cdot$ Yong Song ${ }^{1} \cdot$ Chao Liu $^{1} \cdot$ Anrui He ${ }^{1} \cdot$ Wei Zhao ${ }^{2}$

\begin{abstract}
Given the energy wastage problem of the laminar cooling system of hot rolling, the dynamic optimization scheduling problem of the system under complex intermittent working conditions was investigated to improve the effective utilization of resources. First, combined with production schedules and rolling rhythm times, an On-line Sequential Extreme Learning Machine (OS-ELM) based laminar cooling water consumption prediction model was developed for predicting water demand trends to maintain system supply-demand balance at pump stations. Then, the scheduling instruction optimization model, which considers the system intermittent production conditions and the feedback optimization mechanism, was proposed to reduce the system cooling water overflow and smooth the high cistern level fluctuations. And an adaptive operation scheme optimization model of pumping station based on scheduling instructions was proposed to minimize the total shaft power of pumping station. Finally, the strategies were solved using an improved sparrow search algorithm (ISSA). Experimental simulations demonstrate the effectiveness and supremacy of the proposed method for industrial energy saving in complex intermittent conditions.
\end{abstract}

Key words Industrial energy saving, Laminar cooling, Intermittent production, On-line Sequential Extreme Learning Machine, Improved sparrow search algorithm

\section{Introduction}

The goal of "emission peak and carbon neutrality" [1]

Yong Song

songyong@ustb.edu.cn

Fenjia Wang

ustbwfjhs@163.com

Chao Liu

liuchao2018@ustb.edu.cn

Anrui $\mathrm{He}$

harui_ustb@163.com

Wei Zhao

13841201206@163.com

1 National Engineering Research Center for Advanced Rolling Technology and Intelligent Manufacturing, University of Science and Technology Beijing, 30 Xueyuan Road, Haidian District, Beijing 100083, China

2 Anshan Iron and Steel Group Company Limited, 1 Huangang Road, Tiexi District, Liaoning, 114021, China. has put forward new requirements for energy saving and emission reduction in the manufacturing industry. Building an intelligent and energy-efficient production system has become a key development direction for enterprises. And improving energy utilization by refined energy management is also an inevitable trend in developing industrial energy efficiency. Industrial sites, such as hot rolling laminar cooling systems, are often subject to resource abuse due to complex intermittent production conditions and process requirements. Improving the effective utilization of resources while ensuring product quality has become a pressing issue in the field of industrial energy saving.

Scholars at home and abroad have made many valuable attempts to solve the problem of energy saving and emission reduction in steel industries. Ronghwa et al. [2] analyzed the relationship between energy production and use in equipment, production processes, and each production plant, proposing an integrated management scheme for energy consumption in steel enterprises. Considering the energy consumption interactions of different subsystems, Larsson et al. [3] 
analyzed the difficulty of improving the energy-saving potential of steel mills, proposing a general analysis method for steel mill systems based on process integration models of optimization procedures. Zhang et al. [4] offered low carbon technologies and carbonneutral routes for the steel industry, providing new ideas for energy conservation in the steel industry. It is easy to see that most of the current studies on energy saving and emission reduction in the steel field stay at a relatively macro level and does not provide detailed solutions to the problem of resource wastage caused by complex intermittent working conditions. Therefore, solving the energy consumption problem in laminar cooling systems due to intermittent production conditions requires drawing on research results from other fields.

Sasaki Kento at al. [5] concluded that operating conditions with intermittent, fluctuating, and flexible demands are the leading reasons for supply-demand imbalances in the operation of residential distributed energy grids, using predictive model control to achieve optimal grid operation and effectively reduce operating costs. Ding at al. [6] proposed the principle of building an integrated electricity-hydrogen energy network to solve the problem of new energy electrical energy aggregation and intermittent electrical energy consumption, providing valuable suggestions for decarbonization of the grid system. Zuo at al. [7] developed a parallel combined sensible-latent heat storage unit with intermittent flows based on the intermittent circulation characteristics of the heat transfer fluid, improving the economic efficiency of centralized power generation effectively. Based on the scientific research ideas and methods in the literature mentioned above, we investigated the energy-saving optimal scheduling for laminar cooling water supply pumping stations to solve the resource wastage arising from complex intermittent production conditions.

Due to the intermittent characteristics of hot rolling, it is difficult for pumping stations to meet the frequently changing water demand. Plants generally ensure the cooling quality of their products at the expense of lower resource utilization. Therefore, we proposed the pump station energy-saving optimal scheduling method [8-10].

First, a laminar cooling water consumption prediction model was established to meet the supplydemand balance of the system [11-12]. The prediction model based on time series analysis methods [13] was widely used for urban water consumption prediction. Still, its stability and timeliness cannot meet the demand of laminar cooling systems with complex and variable working conditions. The BP neural network regression prediction model [14-15] is relatively reliable. But it still encounters the problem of long training time and even model collapse in industrial applications due to the increasing amount of data. Therefore, in this paper, OSELM [21-22] was used to predict the water demand trend of pumping stations based on the cooling mechanisms and process specifications [16-20], combined with the production schedule and the actual rolling rhythm time.

Then, based on the intermittent cooling conditions [16-20] and the operation status of the high cistern, a pump station scheduling instruction optimization model with the feedback optimization mechanism was established to minimize the sum of the high cistern water fluctuation and cooling water overflow during the cooling process of strips in a single batch. And the model will update the optimal value of the pump station scheduling instruction in real time according to the high cistern water level and the rolling rhythm.

Finally, a decision variable adaptive pumping station operation scheme optimization model was proposed based on the pump operation characteristics to minimize the total shaft power of the pumping station and significantly reduce the model calculation time. ISSA [23] was used to solve the model.

After optimization, the system can update the optimal operation plan in real time according to the observable status such as rolling rhythm and the measured water level in the high cistern, operating safely and economically while meeting the process production requirements. Experimental calculations at the $1780 \mathrm{~mm}$ plant of the hot rolling mill verified the effectiveness of the proposed method for improving the energy efficiency of the laminar cooling water supply system.

\section{Laminar cooling system}

\subsection{System energy wastage problem}

The laminar cooling system of the hot rolling provides cooling for the strip in accordance with production processes to ensure the quality of strip products, including major equipment such as high cistern, cooling tower, filter tank, water supply pump station and cooling water controller, etc. The system structure is 
shown in Fig. 1.

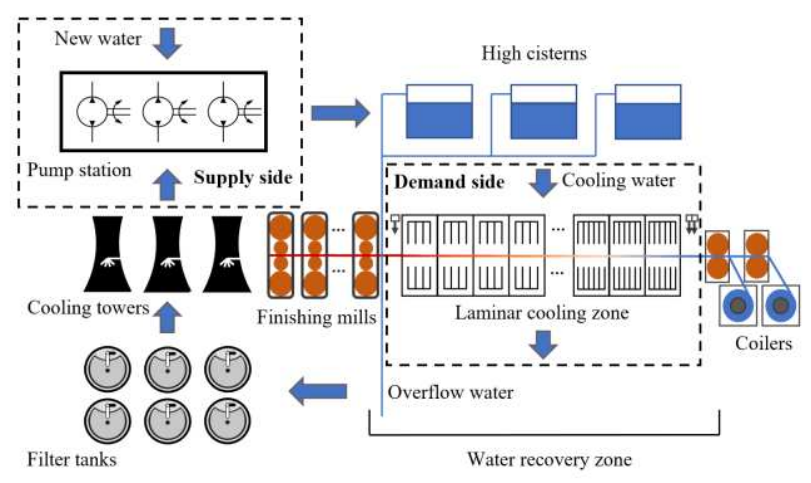

Fig. 1 Structure of laminar cooling system

The system can be divided into two main parts: the supply side and the demand side. The demand side provides real-time adjustment of the cooling header valve statuses to control the strip coiling temperature precisely by the laminar cooling calculation model [24]. In contrast, the supply side provides pressure-stabilized cooling water to the demand side by the pump station and the high cistern.

As shown in Figure 2, the laminar flow cooling calculation model, using a segmented cooling mechanism, adjusts the strip cooling strategy according to the measured feedback value of the coiling temperature to achieve accurate real-time control of the strip cooling.

Theoretically, it is necessary to plan the operation of the water supply system based on the laminar cooling water demands. However, due to the complexity of the cooling control mechanism described above, the variability of working conditions, and the diversity of hot-rolled strip products, water consumptions on the demand side are difficult to calculate using traditional methods.

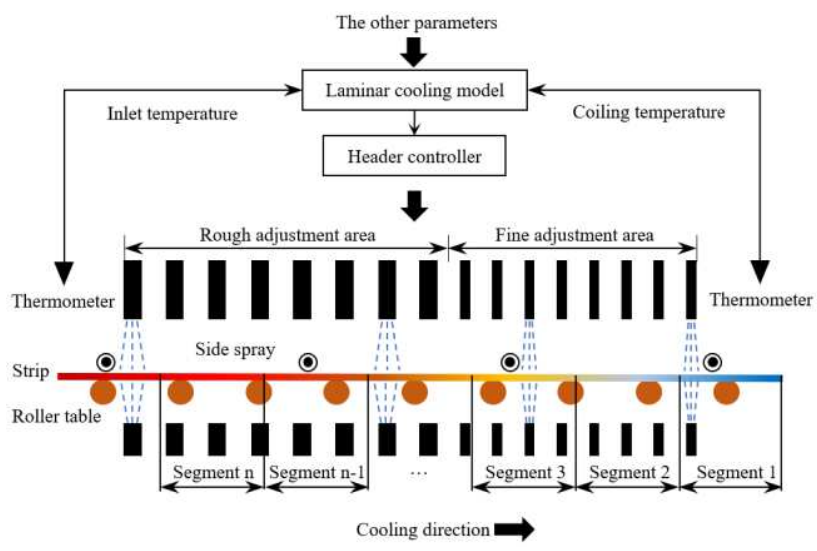

Fig. 2 Laminar flow cooling control mechanism

2.1 Analysis of complex intermittent production conditions
The valves in demand sides are open when the strip is cooling. In contrast, valves are closed between the end of strip cooling and the entry of the next strip into the cooling zone. For ease of understanding, the periods for opening and closing the header valves are defined as the cooling period and the cooling interval, respectively. And they are collectively referred to as the rolling rhythm.

In laminar cooling systems, the high cistern is similar to an energy storage device, providing cooling water with a certain pressure to the demand side. Laminar cooling processes require a slight water level fluctuation in the high cistern, generally $\pm 200 \mathrm{~mm}$. The volume formed by the upper and lower limits of the water level is the effective volume.

Water level fluctuations in the high cistern show the following pattern with rolling rhythm: during the cooling period, the water level keeps dropping because the cooling water consumption rate is greater than the pumping station water supply rate; during the cooling interval, the pumping station supplies water to the high cistern, the water level gradually rises to the initial state of cooling because the water collection valves are closed.

The optimal operation of the high cistern is that the water consumption of laminar cooling processes during the cooling period is the sum of the water supply of the pumping station and the effective volume of the high cistern, and the water supply of the pumping station is equal to the effective volume during the cooling interval. In other words, the high cistern makes its water level meet the process requirements and does not produce the cooling water overflow. Expressed in the formula as:

$Q_{1} t_{1}=Q t_{1}+Q t_{2}$

$Q t_{2}=V_{\mathrm{m}}$

Where $Q_{1}$ is the laminar cooling process water consumption rate, $t_{1}$ is the strip laminar cooling time, $Q$ is the pump water supply rate, $t_{2}$ is the strip cooling interval time, $V_{\mathrm{m}}$ is the effective volume of the high cistern.

In optimal scheduling, the supply-demand balance of the system is necessary to maintain the optimal operation of high cisterns. The combination of optimizing pumping station dispatching instructions based on the accurate prediction of water demand changing trends on the supply side and formulating a scientific pumping 
station operation plan by combining with rolling rhythm time and pump operation characteristics is an effective solution to improve the economy and stability of the system.

\section{Water consumption prediction model based on OS-ELM}

Accurately predicting the consumption of laminar cooling water is key to maintaining a supply-demand balance in the laminar cooling system. Based on the analysis of laminar flow cooling characteristics in Section 2.1, we applied machine learning algorithms for predictive modeling in this paper.

\subsection{Model preprocessing}

Laminar cooling includes heat transfer in forced convection heat transfer, heat conduction between rolls, convection heat transfer by air cooling, and thermal radiation heat transfer. Its water consumption is mainly influenced by the parameters of production processes. Combined with the cooling heat transfer mechanism [1620], we selected the process parameters shown in Table 1 as the factors influencing laminar cooling water consumption in this paper.

Table 1 The influence factors of water consumption

\begin{tabular}{ll}
\hline Parameter & Symbol \\
\hline Strip length & $L$ \\
Strip width & $W$ \\
Strip thickness & $H$ \\
Strip cooling speed & $v$ \\
Finishing temperature & $T_{\mathrm{F}}$ \\
Coiling temperature & $T_{\mathrm{C}}$ \\
Cooling water temperature & $T_{\mathrm{W}}$ \\
Steel grade & $G$ \\
\hline
\end{tabular}

According to the cooling mechanism shown in Fig.

2, the laminar cooling history data for each strip is combined from the production records of each segmented strip. To accurately describe the overall cooling effect of individual strips, this paper first extracted the data of the influence factors in table 1 from the historical data of each segmented strip, generating the laminar cooling water consumption sample data from the average of each influence factor data.

The cooling water flow in the historical data is recorded as the number of header switches, and the following processing is required:

$C_{\mathrm{w}}=\left(R_{\text {mean }} V_{\mathrm{R}}+F_{\text {mean }} V_{\mathrm{F}}\right) t_{\mathrm{C}}$
Where $C_{\mathrm{W}}$ is the cooling water consumption, $R_{\text {mean }}$ is the average number of opening headers in the coarse-tuning area, $V_{\mathrm{R}}$ is the unit header flow rate in the coarse-tuning area, $F_{\text {mean }}$ is the average number of opening headers in the fine-tuning area, $V_{\mathrm{F}}$ is the unit header flow rate in the fine-tuning area, and $t_{\mathrm{C}}$ is the cooling time.

Then the samples were cleaned and standardized.

\subsection{Predictive modeling}

Industrial applications require fast and accurate calculation models. OS-ELM updates the network parameters according to the current data during the training process, without retraining the historical data. The algorithm has fast calculation speed and strong generalization capability, avoiding the problems of long training time or even model collapse that easily occur in increasingly large datasets effectively.

The algorithm is divided into two phases: initial training and sequential learning. In the initial training phase, the initial output weight $\beta_{0}$ is updated by $N_{0}$ random training samples of laminar cooling water consumption, where $N_{0}$ needs to be larger than the number of nodes $L$ of the hidden layer. The steps are as follows:

$$
\begin{aligned}
& D=\left\{\left(x_{i}, t_{i}\right) \mid x_{i} \in R^{n}, t_{i} \in R^{m}\right\} \\
& \sum_{i=1}^{L} \boldsymbol{\beta}_{i} g\left(w_{i} x_{j}+b_{i}\right)=t_{j}, j=1,2, \mathrm{~L}, N_{0}
\end{aligned}
$$

where $\mathrm{D}$ is the training sample set. $w_{i}=\left[w_{i 1}, w_{i 2}, \ldots, w_{i n}\right]^{\mathrm{T}}$, is the random weights of the input layer and the hidden layer nodes. $b_{i}$ is the random bias of the hidden layer nodes. $\boldsymbol{\beta}_{i}=\left[\beta_{i 1}, \beta_{i 2}, \ldots, \beta_{i m}\right]^{\mathrm{T}}$ are the weights of the hidden layer nodes and the output layer.

According to Eq. 5, the output matrix of the hidden layer can be expressed as:

$$
\boldsymbol{H}_{0}=\left[\begin{array}{ccc}
g\left(w_{1}, b_{1}, x_{1}\right) & \mathrm{L} & g\left(w_{n}, b_{L}, x_{1}\right) \\
\mathrm{K} & \mathrm{L} & \mathrm{L} \\
g\left(w_{1}, b_{1}, x_{N_{0}}\right) & \mathrm{L} & g\left(w_{n}, b_{L}, x_{N_{0}}\right)
\end{array}\right]_{N_{0} \times L}
$$

Then the output matrix of the output layer can be expressed as:

$$
\boldsymbol{H}_{0} \boldsymbol{\beta}=\boldsymbol{T}_{0}
$$

Where $\boldsymbol{\beta}=\left[\beta_{1}, \beta_{2}, \ldots, \beta_{L}\right]^{\mathrm{T}}, \boldsymbol{T}_{0}=\left[t_{1}, t_{2}, \mathrm{~L}, t_{N_{0}}\right]^{\mathrm{T}}$.

OS-ELM strives for the minimum error between the predicted and actual values, as shown in equation (8). $\min \left\|\boldsymbol{H}_{0} \boldsymbol{\beta}-\boldsymbol{T}_{0}\right\|$

Based on the generalized inverse of the matrix, the 
initial output weight $\beta_{0}$ can be calculated.

$\beta_{0}=H^{+} T_{0}$

Where $\boldsymbol{H}^{+}=\left(\boldsymbol{H}_{0}{ }^{\mathrm{T}} \boldsymbol{H}_{0}\right)^{-1} \boldsymbol{H}_{0}{ }^{\mathrm{T}}$, is the generalized inverse matrix of $H_{0}$.

The sequential learning phase updates the network output weights $\beta$ by continuous iterative calculations. Let $\boldsymbol{P}_{0}=\left(\boldsymbol{H}_{0}{ }^{\mathrm{T}} \boldsymbol{H}_{0}\right)^{-1}$. Assuming that the hidden layer output of the $\mathrm{k}+1$ th learning is $H_{k+1}$, then $P_{k+1}$ and $\beta_{k+1}$ can be expressed as:

$\boldsymbol{P}_{k+1}=\boldsymbol{P}_{k}-\boldsymbol{P}_{k} \boldsymbol{H}_{k+1}^{\mathrm{T}}\left(\boldsymbol{I}+\boldsymbol{H}_{k+1} \boldsymbol{P}_{k} \boldsymbol{H}_{k+1}^{\mathrm{T}}\right)^{-1} \boldsymbol{H}_{k+1} \boldsymbol{P}_{k}$

$\boldsymbol{\beta}_{k+1}=\boldsymbol{\beta}_{k}+\boldsymbol{P}_{k+1} \boldsymbol{H}_{k+1}^{\mathrm{T}}\left(\boldsymbol{T}_{k+1}-\boldsymbol{H}_{k+1} \boldsymbol{\beta}_{k}\right)$

In the modeling process, the Sigmoid function and the Relu function are chosen as the activation functions of the hidden and output layers, respectively, to enhance the nonlinear expression capability of the model. The number of samples $N_{0}$ in the initial training phase was chosen as $2 L$, and the optimal $L$ is 15 according to the cross-validation data test. The model structure is shown in Fig. 3.

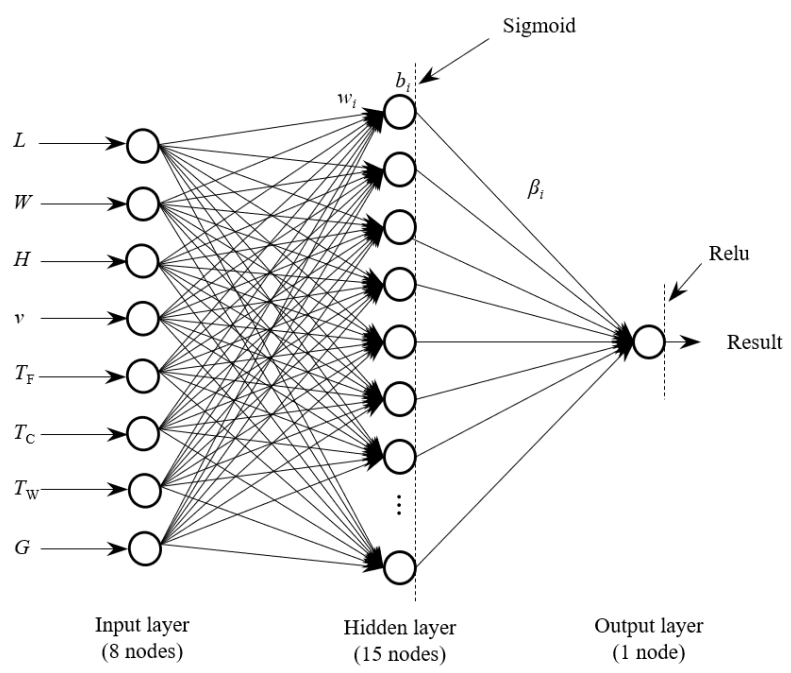

Fig. 3 Structure of the prediction model

\section{Optimal scheduling modeling}

4.1 Optimization model of pump station scheduling instructions

For the complex intermittent production conditions of the laminar cooling system, the pump station scheduling instructions are optimized to keep the optimal operation of the high cistern based on the predicted trend of water consumption rate on the demand side of the system.

\subsubsection{Optimization modeling}

Combined with the water level changing patterns of the high cistern under intermittent production conditions presented in section 2.2, this paper discretized the water level data, taking water level $h_{i}$ before the start of each strip cooling period and water level $h_{1, i}$ after the end of the strip cooling period as the water level characteristic points for water supply optimization work. The water level characteristic points can be expressed as:

$h_{i}= \begin{cases}h_{\max }, & h_{i}>h_{\max } \\ h_{i}, & h_{i} \leq h_{\max }\end{cases}$
$h_{1, i}=h_{i}+\left(Q_{i}-Q_{1, i}\right) t_{1, i} / A$
$h_{i+1}=h_{1, i}+Q_{i} \times t_{2, i} / A$

Where $i$ is the number of strips. $h_{\max }$ and $h_{\min }$ are the upper and lower limits of the high cistern water level, respectively. $A$ is the bottom area of high cisterns, $Q_{1, i}$ is the water consumption rate of laminar cooling processes, $t_{1, i}$ is the strip cooling time, $t_{2, i}$ is the cooling interval time.

In addition, considering the standard range of high tank water level processes, the model applies a lowerlimit penalty term of water levels and an overflow flow penalty term in the objective function, as shown in Eq. 13.

$$
\min \left\{C_{1}+C_{2}+Y L_{1}+Y L_{2}\right\}
$$

Where $C_{1}$ is the sum of the water level $h_{i}$ change in high cistern during the cooling of a single batch of strips, $Y L_{1}$ is the total overflow of the high cistern during operation, $C_{2}$ is the lower-limit penalty term of water levels, $Y L_{2}$ is the overflow penalty term.

Term $C_{1}$ can keep the cooling water pressure stable, as shown in Eq. 14.

$$
C_{1}=\sum_{i=1}^{m}\left(\left|h_{i+1}-h_{i}\right|\right)
$$

where $m$ is the number of strips in the current rolling batch.

Term $Y L_{1}$ can reduce the high cistern overflow during the rolling cycle, as shown in Eq. 15.

$$
\begin{aligned}
& Y L_{1}=\sum_{i=1}^{m} Y_{i} \\
& Y_{i}=\left\{\begin{array}{cc}
Q_{i} t_{2, i}-\left(h_{\text {max }}-h_{1, i}\right) A, & Q_{i} t_{2, i}-\left(h_{\text {max }}-h_{1, i}\right) A>0 \\
0 \quad, Q_{i} t_{2, i}-\left(h_{\text {max }}-h_{1, i}\right) A \leq 0
\end{array}\right.
\end{aligned}
$$

Where $Y_{i}$ is the cooling water overflow rate of the $i$ th strip.

Term $C_{2}$ prevents the water level from falling below the process requirement, as shown in Eq. 16.

$$
\begin{aligned}
C_{2} & =\sum_{i=1}^{m} f_{i} \\
f_{i} & = \begin{cases}\varphi \times\left|h_{1, i}-h_{\min }\right|, & , h_{1, i}<h_{\text {min }} \\
0 & , h_{1, i} \geq h_{\text {min }}\end{cases}
\end{aligned}
$$


The overflow penalty term $Y L_{2}$ prevents the high cistern from being filled for a long time, as shown in Eq. 17.

$$
Y L_{2}=\sum_{i=1}^{m} \gamma \times Y_{i}
$$

In Eq. 16 and 17, $\varphi$ and $\gamma$ are penalty coefficients.

According to the water level Changing patterns of the high cistern described above, the model constraints can be simplified to the upper limit of the water level $h_{i}$ and the lower limit of the water level $h_{1, i}$, as shown in Eq. 18.

$$
\left\{\begin{array}{l}
h_{i} \leq h_{\text {max }} \\
h_{1, i} \geq h_{\text {min }}
\end{array}\right.
$$

\subsubsection{Feedback optimization mechanism}

Due to the errors in the prediction model of water consumption and the deviations between the dispatch values of water supply speeds in pump stations and the actual values, the pump station may still experience water level overrun and cooling water overflow when executing the predetermined dispatching instruction of water supply speeds. Therefore, it is necessary to provide real-time feedback optimizations for pump station scheduling instructions by combining actual rolling rhythms and measured water level values of high cisterns.

The feedback optimization mechanism requires precalculations of water levels $h_{1, i}$ and $h_{i+1}$, as shown in Eq. 19-21.

$V_{i}=p_{i} / t_{1, i}$

$h_{1, i}=h_{i}-\left(V_{i}-Q_{i}\right) t_{1, i} / A$

$h_{i+1}=h_{1, i}+Q_{i} t_{2, i} / A$

Where $V_{i}$ is the strip water consumption rate, $p_{i}$ is the predicted value of strip laminar water consumption.

When the predicted water level exceeds the limit, the water supply rate in the scheduling instruction is corrected, as shown in Eq. 22 and Eq. 23.

$$
\begin{aligned}
& Q Z_{1, i}=\left\{\begin{array}{cc}
V_{i}-\left(h_{i}-h_{\min }\right) \times A / t_{1, i}, & h_{1, i}<h_{\text {min }} \\
Q_{i} & , h_{1, i} \geq h_{\text {min }}
\end{array}\right. \\
& Q Z_{i}=\left\{\begin{array}{cc}
\left(h_{\text {max }}-h_{1, i}\right) \times A / t_{2, i}, & h_{1+i} \geq h_{\text {max }} \\
Q_{i} & , h_{1+i}<h_{\text {max }}
\end{array}\right.
\end{aligned}
$$

Where $Q Z_{1, i}$ is the correction value of water supply speeds for cooling periods, $Q Z_{i}$ is the correction value for the cooling interval.

The technical route of the feedback optimization mechanism is shown in Fig. 4.

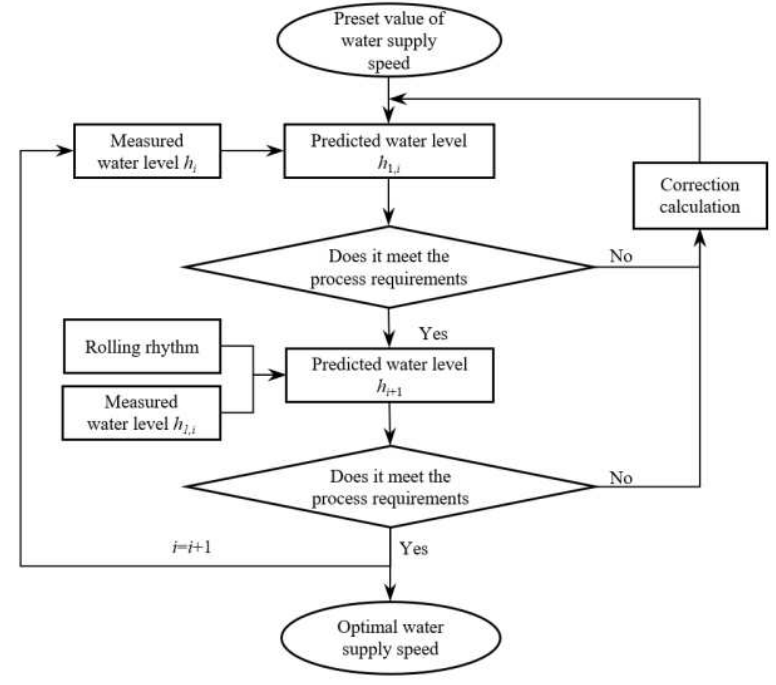

Fig. 4 Feedback optimization mechanism technical route

\subsection{Pump station operation scheme Optimization model}

\subsubsection{Objective function}

Considering the operation efficiency and characteristics of the pump, we proposed an optimization model of operation scheme to achieve efficient and stable operation of the pump station. The variable frequency pump characteristics follow the law of pump speed regulation [26]:

$\frac{Q_{j}}{Q_{0}}=\frac{n_{j}}{n_{0}}=S_{j}, \frac{H_{j}}{H_{0}}=\left(\frac{n_{j}}{n_{0}}\right)^{2}=S_{j}^{2}, \frac{N_{j}}{N_{0}}=\left(\frac{n_{j}}{n_{0}}\right)^{3}=S_{j}^{3}$

Where $Q_{0}$ is the rated water supply speed of pumps, $H_{0}$ is the rated head, $N_{0}$ is the rated shaft power, $n_{0}$ is the rated speed of pumps, $n_{j}$ is the working speed, $S_{j}$ is the speed regulation ratio.

The water pump characteristic curve [27] is generally fitted to the following form:

$H_{j}=a_{0, j} S_{j}{ }^{2}+a_{1, j} Q_{j}{ }^{2}$
$N_{j}=b_{0, j} S_{j}{ }^{3}+b_{1, j} S_{j}^{2} Q_{j}+b_{2, j} S_{j} Q_{j}{ }^{2}$
$\eta_{j}=c_{0, j}+c_{1, j}\left(Q_{j} / S_{j}\right)+c_{2, j}\left(Q_{j} / S_{j}\right)^{2}$

Where $j$ is the pump number, $H_{j}$ is the pump head, $N_{j}$ is the pump shaft power, $\eta_{j}$ is the pump operating efficiency. $a, b$, and $c$ are the pump characteristics parameters, the subscript is the parameter number. $\mathrm{Q}$ is the pump water supply speed. When $S_{j}$ is constant to 1 , the pump is a frequency pump.

The objective function was established to minimize the total shaft power of the pumping station. Assuming that the inverter and frequency pumps operate in parallel at the pumping station, according to Eq. 25, the objective function can be expressed in the following form: 


$$
\begin{aligned}
& \min \left\{\sum_{j=1}^{n} w_{j}\left(b_{0, j}+b_{1, j} Q_{j}+b_{2, j} Q_{j}^{2}\right)+\right. \\
& \left.\sum_{j=n}^{n+k} w_{j}\left(b_{0, j} S_{j}^{3}+b_{1, j} S_{j}^{2} Q_{j}+b_{2, j} S_{j} Q_{j}^{2}\right)\right\}
\end{aligned}
$$

Where $n$ is the number of frequency pumps, $k$ is the number of inverter pumps. $w_{j}$ is the $0-1$ decision variable, 0 means the pump is off, and 1 represents the pump is on.

However, Eq. 26 contains a total of $(2 \mathrm{n}+3 \mathrm{k})$ decision variables such as $w_{j}, S_{j}$, and $Q_{j}$, which are of high complexity. In the actual production, the pump station scheduling instruction is $\left(Q_{s t}, H\right)$, where $Q_{s t}$ is the total water supply flow of the pumping station. Since the parallel operation of pumps requires a consistent head, we replaced the decision variable $Q_{j}$ with the head $H$ based on Eq. 25 to reduce the complexity of the model effectively. Let $Q_{j}=q_{j}$, as shown in Eq. 5 .

$$
q_{j}=\sqrt{\left(H_{j}-a_{0, j} S_{j}^{2}\right) / a_{1, j}}
$$

According to Eq. 26 and 27, the objective function can be expressed as follows:

$$
\begin{aligned}
& \min \left\{\sum_{j=1}^{n} w_{j}\left(b_{0, j}+b_{1, j} q_{j}+b_{2, j} q_{j}{ }^{2}\right)+\right. \\
& \left.\sum_{j=n}^{n+k} w_{j}\left(b_{0, j} S_{j}{ }^{3}+b_{1, j} S_{j}{ }^{2} q_{j}+b_{2, j} S_{j} q_{j}{ }^{2}\right)\right\}
\end{aligned}
$$

\subsubsection{External penalty items}

The speed regulation ratio was set within a safe range to ensure the safe operation of the pump.

$$
S_{j} \in\left[S_{\min }, 1\right]
$$

The total flow constraint requires that the entire water supply of the pump station be equal to the water supply values of the dispatch instruction. It can be expressed as:

$Q_{s t}=\sum_{j=1}^{n} w_{j} Q_{j}+\sum_{j=n}^{n+k} w_{j} Q_{j}$

To ensure efficient operations of the pump, the model sets a high-efficiency interval constraint with a critical efficiency of $80 \%$. According to the characteristic curve of the pump, the high-efficiency interval constraint can be expressed as Fig. 5.

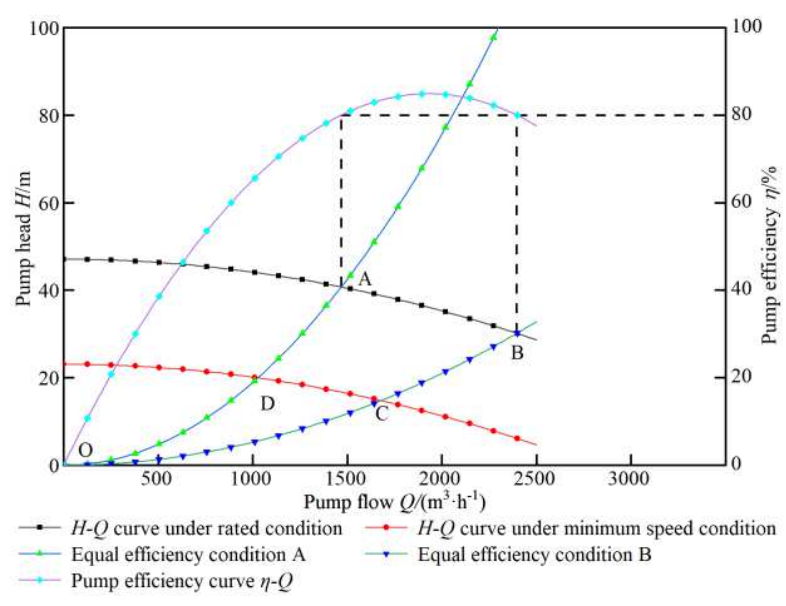

Fig. 5 Pump efficiency interval constraint

In the figure, $A B$ is the head-flow curve of the inverter pump at rated speed, $\mathrm{CD}$ is the head-flow curve of the pump at the lowest speed ratio working condition, $\mathrm{OA}$ and $\mathrm{OB}$ are the equal-efficiency working condition curves of pumps. And the high-efficiency working interval of the pump is the range surrounded by $A B C D$, using the equation formulas as:

For inverter pumps:

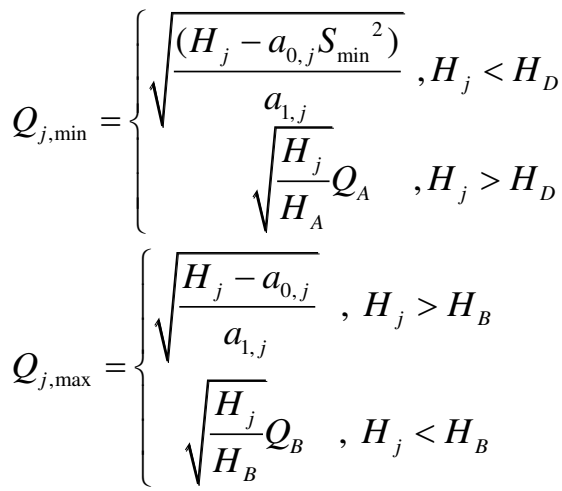

For frequency pumps:

$Q_{j, \text { min }}=Q_{A}$

$Q_{j, \text { max }}=Q_{B}$

To improve the accuracy and solution speed of the model, we simplified the model by applying the highefficiency interval penalty term $T F_{2}$ and the total flow penalty term $\mathrm{TF}_{3}$ to the objective function.

$$
T F_{2, j}= \begin{cases}Q_{j, \text { min }}-\sqrt{\frac{\left(H_{j}-a_{0, j} S_{j}^{2}\right)}{a_{1, j}}} & , Q_{j}<Q_{j, \text { min }} \\ 0 & , Q_{j, \text { min }}<Q_{j}<Q_{j, \text { max }} \\ \sqrt{\frac{\left(H_{j}-a_{0, j} S_{j}^{2}\right)}{a_{1, j}}}-Q_{j, \text { max }} & , Q_{j}>Q_{j, \text { max }}\end{cases}
$$




$$
T F_{3}=\left|Q_{s t}-\sum_{j=1}^{n+k} w_{j} Q_{j}\right|
$$

Then the new objective function can be expressed as: $\min \left\{T F_{1}+\varepsilon\left(T F_{2}+T F_{3}\right)\right\}$

where $\varepsilon$ is the penalty factor.

\subsubsection{Adaptive selection mechanism for decision variables}

The number of decision variables has a significant impact on the speed of the optimization model calculation. In most cases, pump stations do not need all pumps to be on during operation. On the other hand, the optimization model does not need to calculate the operating scheme of the closed pump. Therefore, to reduce the calculation time of the model effectively, we developed an adaptive selection mechanism for decision variables based on scheduling instructions in this paper. The steps are as follows:

In the first step, we set an operation rule that prioritizes inverter frequency pumps over frequency pumps in the operation sequence.

In the second step, the scheduling instruction $\left(Q_{s t}, H\right)$ is substituted into Eq. 31-33 to find the upper and lower limits of the pump water supply speed.

In the third step, the decision variables are selected. Determine whether $Q_{s t}$ is within the interval of $\left(Q_{\min }\right.$, $\left.k Q_{\max }\right)$. Where $\mathrm{k}$ is the number of inverter pumps, $Q_{\min }$ and $Q_{\max }$ are the upper and lower limits of the water supply speed of inverter pumps, respectively. When $Q_{s t}$ is in this interval, the decision variables are the on/off state and the speed ratio of inverter pumps, with a total quantity of $2 k$. Otherwise, the decision variables are the on/off state of the inverter and frequency pumps and the speed regulation ratio of inverter pumps, with a total quantity of $(n+2 k)$.

In the fourth step, the decision variables are applied to the optimization model.

\subsection{Optimal scheduling model solving}

The objective function of the pump station scheduling instruction optimization model contains two factors: the water level of high cisterns and cooling water overflow.

Considering the effects of parameter magnitudes and model optimization weights, we applied the conversion coefficient $\theta$ and the weighting coefficient $\beta$ to Eq. 13 . The final model is shown in Eq. (37). $\min \left\{\beta\left[\theta\left(C_{1}+C_{2}\right)\right]+(1-\beta)\left(Y L_{1}+Y L_{2}\right)\right\}$

In the objective function of the pump station operation scheme optimization model, the term $T F_{1}$ and $T F_{3}$ have different magnitudes, so a conversion factor $\delta$ was added to Eq. 36, as shown in Eq. 38 .

$\min \left\{T F_{1}+\varepsilon \delta\left(T F_{2}+T F_{3}\right)\right\}$

In addition, in Eq. 28, the product of 0-1 variables with other variables was linearized by the big-M [28] method. And the quadratic term of the water supply and the cubic term of the speed regulation ratio are linearized by introducing cone constraints and auxiliary variables [29].

The optimal scheduling pattern of the laminar cooling water supply pumping station is shown in Figure 6. The prediction model of laminar cooling water consumption was used to calculate water consumption trends during the current rolling cycle. Considering the predicted laminar cooling water consumption, complex intermittent system conditions, and actual tank levels, the optimization model of pump station scheduling instructions can keep the system in a supply-demand balance while stabilizing high cistern level fluctuations and reducing cooling water overflows. Combining with the pump operation characteristics and constraints, the optimization model of pump station operation schemes can minimize the total shaft power of the pump station during the rolling cycle, reducing the model solution time by using an adaptive selection mechanism of decision variables. The three models are coupled to achieve energy-efficient operation of the pumping station finally. 


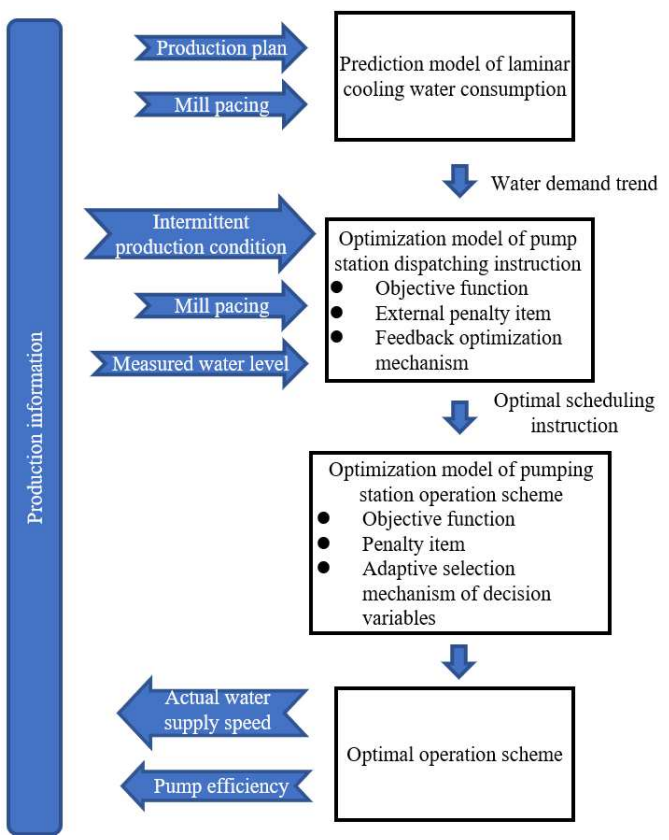

Fig. 6 Optimized scheduling of laminar cooling water supply pumping station

The solution method for the optimal scheduling model is the SSA [23]. And the algorithm was improved according to the characteristics of the scheduling problem.

When the search individuals exceed the limit, SSA will do the following:

$$
\begin{aligned}
& X=\left\{\left(X_{i}\right) \mid X_{i} \in R^{d}\right\} \\
& X_{i}=\left\{\begin{array}{l}
\mathrm{Lb}_{i}, X_{i}<\mathrm{Lb}_{i} \\
\mathrm{Ub}_{i}, X_{i}>\mathrm{Ub}_{i}
\end{array}\right.
\end{aligned}
$$

Where $X$ is an individual sparrow with $d$ variables, $X_{i}$ denotes the parameter in the individual, $\mathrm{Ub}_{i}$ and $\mathrm{Lb}_{i}$ denote the upper and lower limits of the parameter, respectively.

This boundary treatment is not suitable for solving the pumping station operation scheme, making the decision variables aggregate at the boundary during the test, with an undesirable success rate of the model solution. Eq. 40 is improved for the above problem, as shown in Eq. 41.

$$
X_{i}=\mathrm{Lb}_{i}+\operatorname{rand}\left(\mathrm{Ub}_{i}-\mathrm{Lb}_{i}\right), X_{i}<\mathrm{Lb}_{i} \cup X_{i}>\mathrm{Ub}_{i}
$$

In addition, SSA treats the parameters as continuous variables during iteration, while the on/off state of the pump station in the operational scheme optimization model is a $0-1$ decision variable. Therefore, this paper developed a decision variable encoder that efficiently converts continuous variables into $0-1$ decision variables during algorithm seeking reducing energy consumption for pump start and stops by sorting the variables according to the pump type, as shown in Fig. 7.

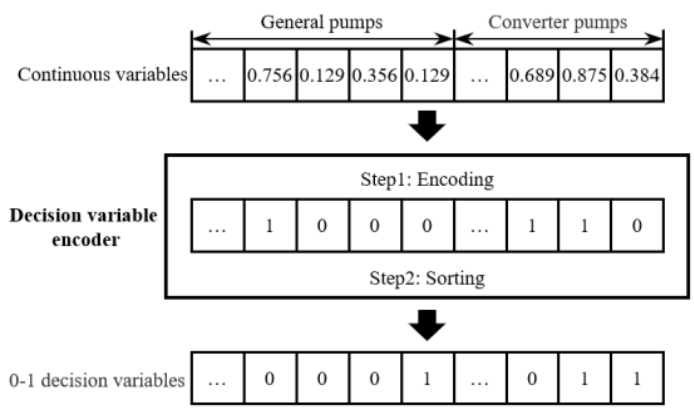

Fig. 7 Decision variable encoder

\section{Algorithm validation}

Simulation tests of the proposed method were carried out on a laminar flow cooling system in a $1780 \mathrm{~mm}$ hot rolling line, with a scheduling cycle of 1 hour and 50 minutes, during which 35 coils of Q235B(C) strip were produced. The pumping station pump parameters are shown in Table 2, and the system model parameters are

\begin{tabular}{|c|c|c|}
\hline \multicolumn{2}{|c|}{ No. Type } & Characteristic curve function \\
\hline $1-4$ & $\begin{array}{c}\text { 24SH19A } \\
\text { (General } \\
\text { pump) }\end{array}$ & $\begin{array}{l}H=39.5-1.507 \times 10^{-6} Q^{2} \\
N=-2.753 \times 10^{-5} Q^{2}+0.1606 Q+8.215 \\
\eta=-9.958 \times 10^{-6} Q^{2}+0.05949 Q-0.01379\end{array}$ \\
\hline $5-7$ & $\begin{array}{c}24 \mathrm{SH} 13 \\
\text { (Converter } \\
\text { pump) }\end{array}$ & $\begin{array}{l}H=40.78 S^{2}-2.948 \times 10^{-6} Q^{2} \\
N=-3.086 \times 10^{-5} Q^{2} S+0.1715 Q S^{2}+8.482 S^{3} \\
\eta=-2.274 \times 10^{-5} Q^{2} / S^{2}+0.08786 Q / S+0.03534\end{array}$ \\
\hline
\end{tabular}
set as shown in Table 3.

Table 2 Pump operating parameters

Table 3 Parameters of optimal dispatching model

\begin{tabular}{lll}
\hline Type & Parameter & value \\
\hline Technical parameters & $\mathrm{A} / \mathrm{m}^{2}$ & $3 * 100$ \\
of high cistern & $h_{0} / \mathrm{m}$ & 7.6 \\
\hline Process requirements & $h_{\max } / \mathrm{m}$ & 7.7 \\
of water level & $h_{\min } / \mathrm{m}$ & 7.3 \\
\hline Conversion coefficient & $\theta$ & 300 \\
of water level and flow & & \\
\hline Conversion factor of & $\delta$ & 0.0885 \\
flow and shaft power & & 0.6 \\
\hline Weight coefficient & $\beta$ & 300 \\
\hline Penalty coefficient & $\gamma$ & 200 \\
\hline Scheduling instruction & $H / \mathrm{m}$ & 300 \\
\hline$\left(Q_{s t} H\right)$ & & 27 \\
\hline Pump operating & High efficiency & 80 \\
\hline
\end{tabular}


parameters

critical value $/ \%$

$S_{\min }$

0.7

2.1 Validation of water consumption prediction model

One week's historical production data of the production line were selected for model training to ensure the generalization ability of the prediction model, including 1466 training data and 334 test data. Fig. 8 clearly shows that the OS-ELM-based laminar cooling water consumption prediction model proposed in this paper has an absolute error within 6t, with MAPE of 2.06 and RMSE of 2.82 .

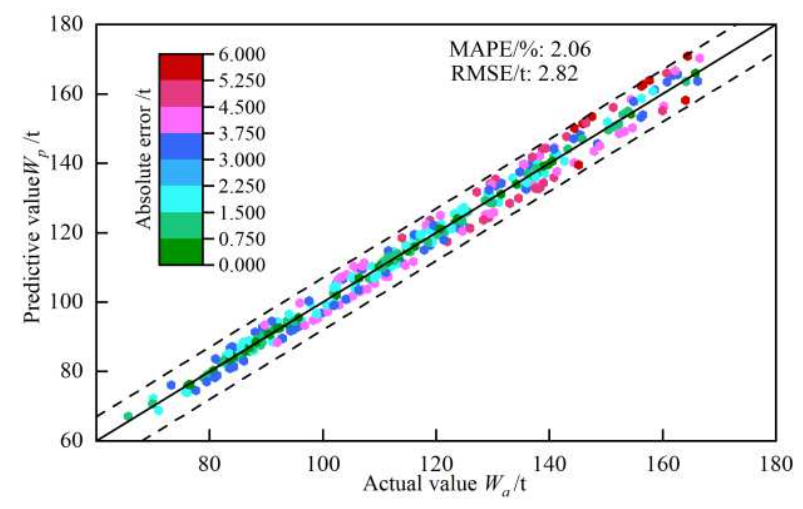

Fig. 8 Model training results

The water consumption prediction results for this scheduling period are shown in Fig. 9. The results show that the model maintains good accuracy in a small range prediction and can be used for the optimization scheduling work of pump stations.

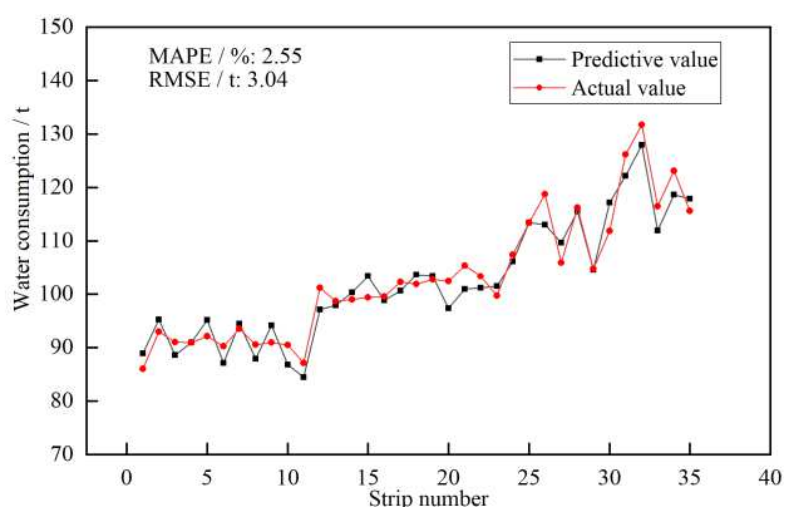

Fig. 9 Prediction results of water consumption during the dispatch period

\subsection{Validation of pump station scheduling instruction optimization model}

According to the rhythm of strip rolling, the cooling period and the cooling interval of the individual strips were defined as two cooling phases. Based on the predicted results of laminar cooling water consumption in the above section and the actual rolling rhythm time, the optimization model of pump station scheduling instructions was verified. The model parameters are shown in Table 3 . The optimization results are shown in Fig. 10 .

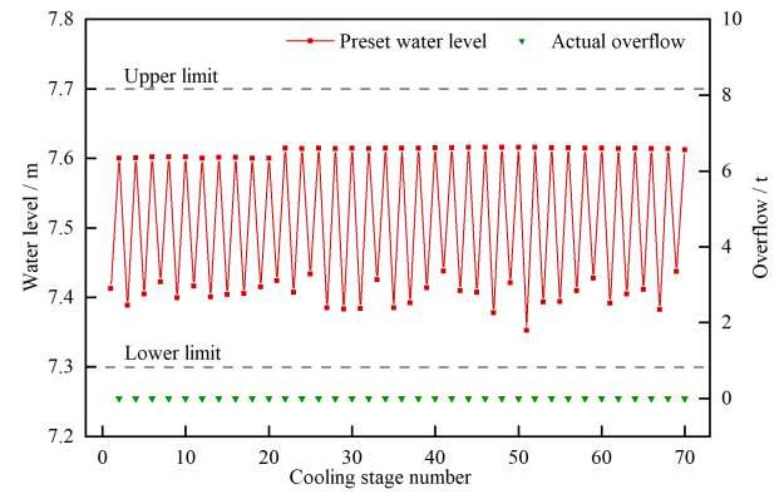

Fig. 10 Optimization effect of pump station scheduling instructions

Fig. 10 clearly shows that when the water supply from the pump station is equal to the optimized scheduling instruction, water levels of the high cistern during the cooling cycle of this batch strips meet the process requirements, and cooling water overflows have disappeared. The model has a good optimization performance and can be used for pumping station operation scheme optimization.

\subsection{Validation of pump station operation scheme} optimization model

\subsubsection{Validation of the feasibility and convergence performance of ISSA}

The feasibility and convergence performance of the ISSA in this paper will be compared against other wellknown algorithms, specifically, the sparrow search algorithm (SSA), the improved particle swarm algorithm (IPSO) [30], the genetic algorithm (GA) [8] and the carnivorous plant search algorithm (CPA) [31].

In this test, all algorithms were simulated for 30 independent runs on the optimal model of the pumping station operation scheme. The parameters of algorithms are summarized as follows:

(1) ISSA and SSA: population size $=50$; Producer probability, $\mathrm{P}=0.2$

(2) IPSO: Population size $=50$; Acceleration factor, $\mathrm{C} 1=2.5$; Acceleration factor, $\mathrm{C} 2=0.5$; Maximum inertia weight, wmax $=0.9$; Minimum inertia weight, 
$\mathrm{Wmin}=0.4$

(3) GA: Population size $=50$; Number of mutation sites $=5$; Crossover probability, $\mathrm{Pc}=0.95$; Probability of variation, $\mathrm{Pm}=0.05$; String length, Sbit $=34$

(4) CPA: nCPlant $=10 ;$ nPrey $=40$; Attraction rate $=$ 0.8 ; group iteration $=2$; Reproduction rate $=1.8$; Growth rate $=2$

Fig. 11 represents the convergence curves of ISSA with other competitive algorithms. It can be seen in the figure that CPA has the fastest convergence rate when using the number of iterations as the evaluation indicator. Still, its Average Best Fit is the worst among all the algorithms. The convergence speed of ISSA is second only to CPA with an insignificant difference, but ISSA outperforms other competitive algorithms in solving Average Best Fit.

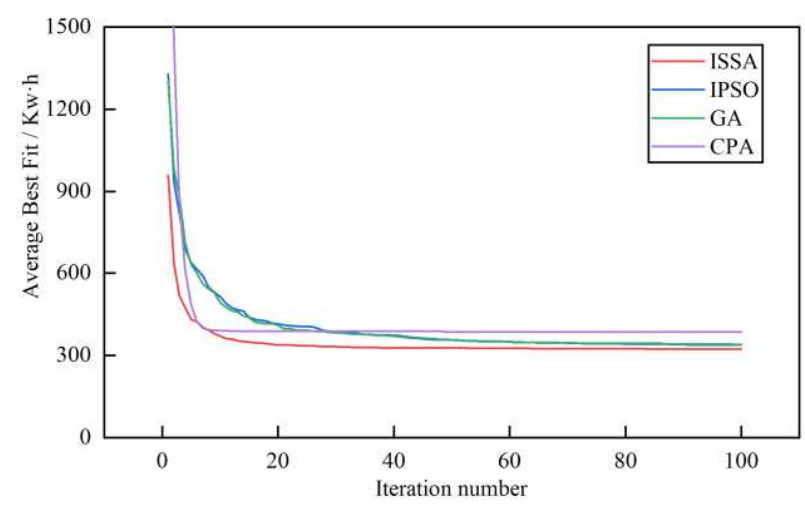

Fig. 11 Convergence curve of ISSA with other competitive algorithms

Table 4 shows the metric results of the algorithm feasibility. After optimization, the dispatching is considered successful when the absolute error between the total water supply speed of the pump station and the dispatching instruction is less than 10 . It can be seen that ISSA has the best performance in terms of success rate and the ability to find the best, but its convergence time is longer than SSA. After analysis, the reason for the longer running time of ISSA than SSA is that the overrun processing algorithm of ISSA is more complex. However, at the same time, ISSA has a significantly higher scheduling success rate than SSA. In addition, CPA also has a superior success rate in finding the best scheme, but it is not suitable for industrial applications due to its long convergence time. ISSA is the most appropriate algorithm for model solving.

Table 4 The percentage improvement of performances between
ISSA with the best rank optimizer among other competitors

\begin{tabular}{lllllll}
\hline & & & & & & Percentage \\
& ISSA & SSA & IPSO & GA & CPA & Improvement \\
& & & & & & $/ \%$ \\
\hline Success & $\mathbf{1 0 0}$ & 72.85 & 85.71 & 88.57 & $\mathbf{9 8 . 5 7}$ & 1.45 \\
rate/\% & & & & & \\
Converge- & & & & & \\
nce time / & $\mathbf{1 2 2 . 4 5}$ & $\mathbf{1 1 1 . 2 1}$ & 141.54 & 117.5 & $2,335.32$ & -10.11 \\
Total & & & & & \\
power/kw & & & & & & \\
\hline
\end{tabular}

\subsubsection{Validation of model scheduling results}

ISSA was applied to solve the model. The scheduling cycle has 70 cooling phases, and the operating schemes of the first 20 cooling stages are selected for demonstration, as shown in Table 5.

Table 5 Operation schemes of pump station in cooling stage 1-20

\begin{tabular}{|c|c|c|c|c|}
\hline $\begin{array}{l}\text { Cooling stages } \\
\text { number }\end{array}$ & $w$ & S5 & S6 & S7 \\
\hline 1 & 0000001 & 0 & 0 & 0.87 \\
\hline 2 & 0000001 & 0 & 0 & 0.85 \\
\hline 3 & 0000001 & 0 & 0 & 0.90 \\
\hline 4 & 0000001 & 0 & 0 & 0.85 \\
\hline 5 & 0000001 & 0 & 0 & 0.86 \\
\hline 6 & 0000001 & 0 & 0 & 0.86 \\
\hline 7 & 0000001 & 0 & 0 & 0.89 \\
\hline 8 & 0000001 & 0 & 0 & 0.85 \\
\hline 9 & 0000001 & 0 & 0 & 0.89 \\
\hline 10 & 0000001 & 0 & 0 & 0.86 \\
\hline 11 & 0000001 & 0 & 0 & 0.87 \\
\hline 12 & 0000001 & 0 & 0 & 0.86 \\
\hline 13 & 0000001 & 0 & 0 & 0.86 \\
\hline 14 & 0000001 & 0 & 0 & 0.87 \\
\hline 15 & 0000001 & 0 & 0 & 0.88 \\
\hline 16 & 0000001 & 0 & 0 & 0.85 \\
\hline 17 & 0000001 & 0 & 0 & 0.88 \\
\hline 18 & 0000001 & 0 & 0 & 0.85 \\
\hline 19 & 0000001 & 0 & 0 & 0.85 \\
\hline 20 & 0000001 & 0 & 0 & 0.87 \\
\hline
\end{tabular}

The pump station operation scheme optimization model was enabled to compare the actual water supply rate of the pump station with the pump station scheduling instructions, and the results are shown in Fig. 12. It can be seen that the actual water supply of each cooling stage is mostly consistent with the dispatching instructions, and the absolute error is less than $2 \mathrm{~m}^{3} \cdot \mathrm{h}^{-1}$. The total water 
supply control performance of the optimized model is desirable.

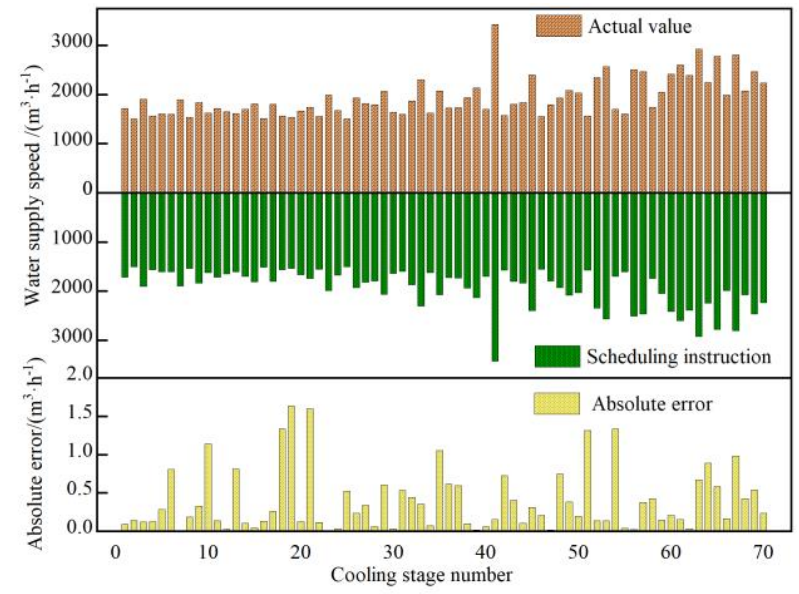

Fig. 12 Total water supply dispatch results of pump stations

Fig. 13 shows the operational efficiency of the pumps during dispatch. It can be seen that most of the pumps operate at efficiencies higher than $80 \%$. The pump efficiencies of the four cooling stages don't meet the requirements, but they were close to $80 \%$. The percentage of high-efficiency operation of water pumps is $95 \%$, and the pump operation efficiency control capability of the optimization model is favorable.

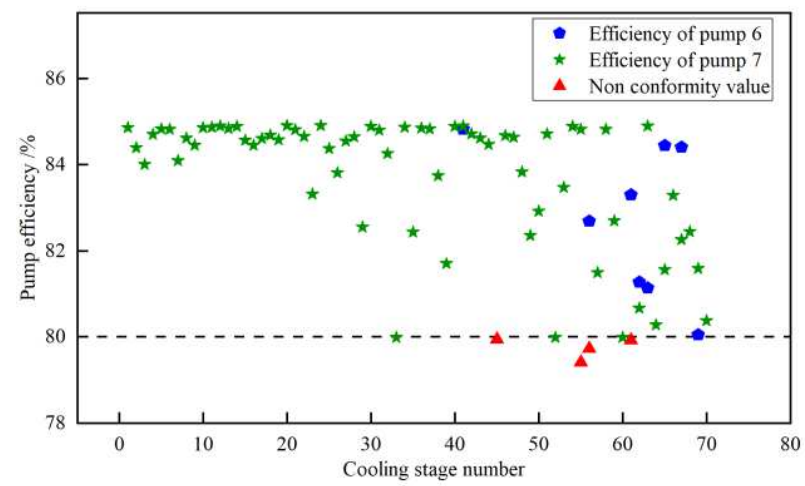

Fig. 13 Statistical chart of water pump operating efficiency

Thirty independent model runs were performed using the ISSA to test the runtime of the pump station operation scheme optimization model with the decision variable adaptive selection mechanism. The average value of the running times is shown in Table 6 . The adaptive selection mechanism of decision variables significantly shortens the convergence time of the model, reducing the average solution time of the pump station operation scheme to $1.009 \mathrm{~s}$ and enhancing the industrial applicability of the model.

Table 6 Convergence time of the model with adaptive decision variables selection mechanism

\begin{tabular}{lll}
\hline & \multicolumn{2}{c}{ Model with selection Percentage } \\
& mechanism & Improvement / \% \\
\hline Converge- & 70.63 & 42.32 \\
nce time / 122.45 & & \\
\hline
\end{tabular}

The energy-saving effect of the model is illustrated as shown in Table 7. It can be seen that the total shaft power of the pumping station is reduced after the optimized scheduling, and the cooling water overflow is significantly reduced, and the resource utilization of the pumping station is effectively improved.

Table 7 Contrast of model scheduling results

\begin{tabular}{|c|c|c|c|}
\hline Parameters & $\begin{array}{l}\text { Before } \\
\text { optimization }\end{array}$ & After optimization & $\begin{array}{l}\text { Percentage } \\
\text { reduction / \% }\end{array}$ \\
\hline $\begin{array}{l}\text { Total power } \\
\text { consumption } / \mathrm{KW}\end{array}$ & 370.87 & 320.43 & 13.6 \\
\hline $\begin{array}{l}\text { Total water supply } \\
/ \mathrm{m}^{3}\end{array}$ & 4027.64 & 3603.32 & 10.54 \\
\hline Overflow $/ \mathrm{m}^{3}$ & 394.57 & 0 & 100 \\
\hline
\end{tabular}

5.4 Discussion of model scheduling results under different operating conditions

Several operating conditions were set up for experiments to determine the effect of different operating conditions on the optimal scheduling of pumping stations.

Condition $1: \mathrm{A}=300 \mathrm{~m}^{2} ; h_{0}=7.7 \mathrm{~m}$

Condition $2: \mathrm{A}=300 \mathrm{~m}^{2} ; h_{0}=7.6 \mathrm{~m}$

Condition $3: \mathrm{A}=300 \mathrm{~m}^{2} ; h_{0}=7.5 \mathrm{~m}$

Fig. 14 shows the actual water level and overflow value of the high cistern for the three operating conditions mentioned above when the pump station scheduling command optimization model is not enabled with the feedback optimization mechanism. It can be seen that the water level control performance of all three conditions is gradually deteriorating with time due to the prediction error of laminar cooling water and the error between the scheduling instructions and the actual water supply speed of the pump. 


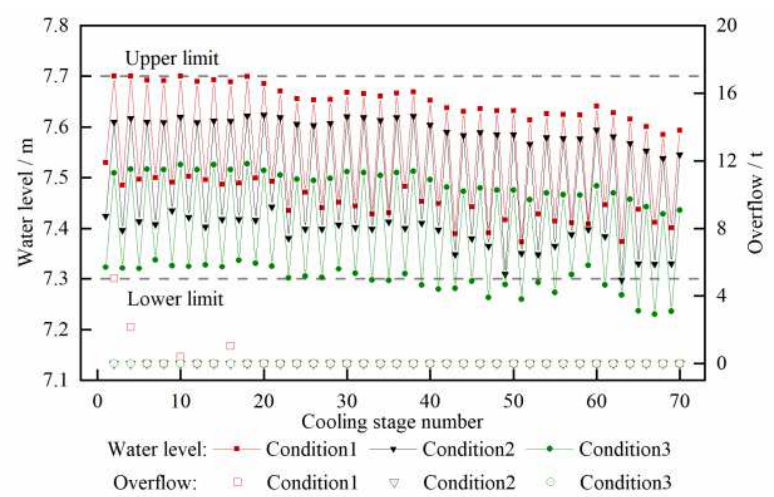

Fig. 14 Water level and overflow of high cistern without applying feedback optimization mechanism

The initial water level of Condition 1 is the upper limit of the high cistern water level. Since the scheduling instruction optimization model restores the water level to the initial state by default after cooling, the water consumption prediction error tends to cause the overflow of cooling water from the high cistern. However, at the same time, there is no case where the water level is below the lower limit.

The initial water level of condition 2 has a specific tolerance range with the upper and lower limits of the water level. Therefore, the prediction model errors do not cause the water level to exceed the standard. During the dispatch cycle, there was no cooling water overflow in condition 2, and water levels below the lower limit rarely occurred.

The initial water level in Condition 3 is close to the lower water level limit. Therefore, later in the dispatch cycle, some water levels of the high cistern will be lower than the lower water level limit. However, at the same time, no cooling water overflow will occur.

Fig. 15 shows the water level and cooling water overflow of the high cistern with the feedback optimization mechanism. It can be seen that the feedback optimization mechanism can effectively solve the problem that the water level control performance gradually deteriorates over time, preventing cooling overflow from occurring in the high cistern while making the water level in the above three conditions all meet the cooling process requirements.

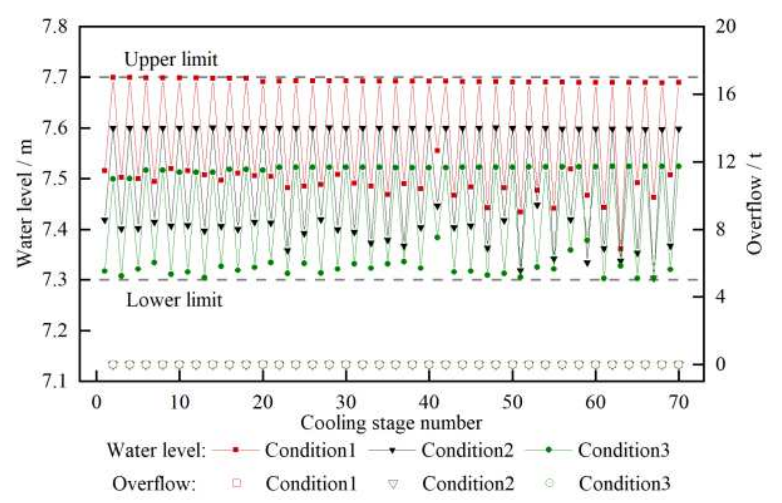

Fig. 15 High cistern water level and overflow when the feedback optimization mechanism is turned on

Several operating conditions were set for the experiment to test the effect of the high cistern effective volume on the optimal scheduling of the pumping station. And the feedback optimization mechanism is turned on in the investigation.

Condition 4: $\mathrm{A}=100 \mathrm{~m}^{2} ; h_{0}=7.6 \mathrm{~m}$

Condition 5: A $=200 \mathrm{~m}^{2} ; h_{0}=7.6 \mathrm{~m}$

Condition 6: $\mathrm{A}=300 \mathrm{~m}^{2} ; h_{0}=7.6 \mathrm{~m}$

Fig. 16 shows the control effect of high cistern water level and overflow for the abovementioned conditions. Due to the small effective volume, the liquid level fluctuates between the upper and lower limits of the process requirements during the dispatch of condition 4. At the same time, cooling water overflow occurs. With the increase of effective volume, the fluctuation range decreases. Therefore, increasing the effective volume of the high cistern can improve the control performance of the water level and overflow for the optimization model.

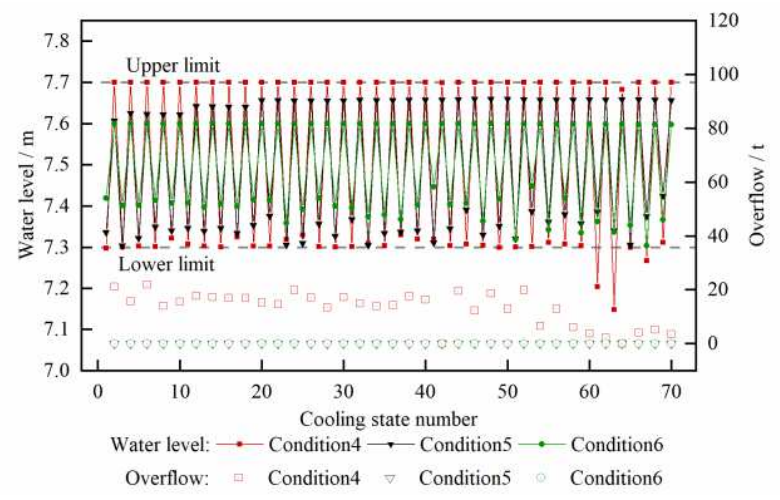

Fig.16 Model control performance of the high cistern water level and overflow with different effective volumes

\section{Conclusion}

A dynamic energy-saving optimal scheduling method for laminar cooling water supply systems was 
proposed in this study, which considers the complex intermittent production conditions, supply-demand balance of the system and process requirements of the high cistern. To validate the robustness of the proposed method, several experiments were conducted. The conclusions are as follows.

(1) The optimal scheduling method proposed in this paper can effectively control the water level and cooling water overflow in high cisterns under the premise of satisfying the supply-demand balance of the laminar cooling water supply system, reducing the total electricity consumption of the pumping station while ensuring the efficient and safe operation of the pumps, effectively improving the utilization rate of the system resources, and providing new ideas for energy saving and emission reduction in industries with complex intermittent working conditions.

(2) Over time, errors of the laminar cooling water usage prediction model and deviations of the actual water supply of the pumping station from the scheduling

\section{Declarations}

Funding This work was financially supported by the National Natural Science Foundation of China (Grant No.52004029). Author Yong Song has received research support from Company Anshan Iron and Steel Group.

Competing interests The authors declare no competing interests.

\section{Availability of data and material}

The authors confirm that the data and material supporting the findings of this work are available within the article.

Code availability Not applicable.

Ethics approval Not applicable.

Consent to participate Not applicable.

Consent for publication This work is approved by all authors for publication.

\section{Authors' contributions}

All authors contributed to the study conception and design. Material preparation, experimental data collection and analysis, and the first draft of the manuscript were performed by Fenjia Wang. The experimental methods and ideas were provided by Yong Song. The accuracy of the model was verified by Chao Liu. The manuscript was revised under the guidance of Anrui He. The constructive suggestions on experiments were provided by Wei Zhao. instructions will gradually reduce the performance of the optimal scheduling model in terms of high cistern water level and overflow control. It can be effectively addressed by adding a feedback optimization mechanism.

(3) The initial water level value and effective volume of the high cistern affect the optimal dispatching capability of the model. The combination of reasonably selecting the initial value of water level according to the demands and scientifically increasing the effective volume of the high cistern will improve the stability of the optimal dispatching of the pump station and the energy utilization rate of the system, reducing the production cost.

Subsequently, we will analyze the forms of resource wastage of other circulating water systems in the hot rolling, conducting joint optimization scheduling studies on the water supply pump stations of each system, improving further the effective utilization of resources in the hot rolling mill.

\section{References}

[1] Guo C X (2021) Carbon neutrality in 2060 will lead to fundamental changes in China's economic system. Journal of Beijing University of Technology (Social Science Edition), 21(05): 64-77

[2] Ronghwa W, Junghsiang Y, Chiayon C (1989) Energy analysis of an integrated steel mill: A process modeling approach. Energy, 14(12):831

[3] Larsson M, Dahl J (2003) Reduction of the specific energy use in an integrated steel plant-The effect of an optimization model. ISIJ International, 43(10):1664

[4] Zhang Q, Cai J J (2021) Energy saving and energy efficiency improvement of iron and steel manufacturing process system Iron and steel, 56 (08): 32-42

[5] D Sasaki Kento, Aki Hirohisa, Ikegami Takashi (2022) Application of model predictive control to grid flexibility provision by distributed energy resources in residential dwellings under uncertainty[J]. Energy, 239(PB):

[6] Ding J, Fang X S, Song Y T, Zheng C (2021) The conception of electricity hydrogen integrated energy power network for new energy transmission in Western China under the background of carbon neutralization. Automation of electric power systems, 1-10 


\section{DOI: 10.7500/aeps20210211002}

[7] Zuo H Y, Zhou Y, Wu M Y, Zeng K (2021) Development and numerical investigation of parallel combined sensiblelatent heat storage unit with intermittent flow for concentrated solar power plants. Renewable Energy, 175:

[8] Wang T, Yang J (2019) Optimal operation model and application of water supply pump station in M City. Water Resources and Power, 37 (7): 141-145

[9] Fang G H, Cao R, Liu Q (2016) Improved genetic algorithm and its application in optimal operation of pumping station. South-to-North Water Transfer and water science and technology, 14 (02): 142-147

[10] Jimmy H. Gutiérrez-Bahamondes, Salgueiro Y, SilvaRubio S A (2019) jHawanet: An Open-Source Project for the Implementation and Assessment of Multi-Objective Evolutionary Algorithms on Water Distribution Networks.

Water, 11(10)

[11] LI J (2017) Study on joint optimal scheduling of pumping station and clear water tank in Sanshan water plant of Wuhu City. Dissertation, Hefei University of Technology

[12] Ding X (2019) Study on optimal scheduling of secondary pumping stations for urban water supply. Dissertation, Chang'an University

[13] Zhang W W, Zhao P W, Wang J C (2019) Application of urban water demand prediction based on long-term and shortterm neural network. Water Purification Technology, 38 (S1): $257-260+286$

[14] He H T, Liu Y B, Paris M, Zhou C D (2021) Nonlinear dynamic model of active magnetic bearing of flywheel energy storage system based on BP neural network. Proceedings of the CSEE: $1-15$.

DOI: 10.13334/j.0258-8013.pcsee.202592.

[15] Huang Y W, Peng D G, Yao J (2021) Ultra-short-term PV power prediction by TD-BP neural network based on SSA and K-means. Acta Energiae Solaris Sinica 42(04): 229-238

[16] He X (2019) Exploration on the application of laminar flow cooling process control method for hot rolled strip steel. Journal of Materials Science \& Technology, (22): 158-159 [17] Yan L J (2017) Hot rolling laminar flow cooling device. CHFI Technology, 2017(04):10-13+65

[18] Li X D (2017) Research and application of post-rolling cooling control method for hot rolled sheet and strip steel under ultra-fast cooling conditions. Dissertation, Northeastern University (China)

[19] Qiu Z S (2017) Study on the evolution of plate shape after rolling of hot rolled strip steel. Dissertation, University of Science and Technology Beijing

[20] Li Z L, Chen D, Yuan G (2019) Research on intelligent control strategy of ultra-fast cooling system for hot rolled strip steel. Steel Rolling, 36(06): 69-73+92

[21] Guo W, Xu T, Tang K M, Yu J J (2017) Online throughsequence transcendental learning machine with generalized regularization and forgetting mechanism. Journal of Control and Decision, 32(02):247-254

[22] Thamizharasu P, Shanmugan S, Sivakumar S (2021) Revealing an OSELM based on traversal tree for higher energy adaptive control using an efficient solar box cooker. Solar Energy,218:

[23] Fu H, Liu H (2022) Improved sparrow search algorithm with multi-strategy fusion and its application. Journal of Control and Decision,37(01):87-96.

DOI: $10.13195 /$ j.kzyjc.2021.0582

[24] Yuan G, Chen D, Kang J, Li ZB, Wang W D (2019) Technology development and application of new generation of controlled rolling and cooling based on ultra-fast cooling for large hot strip rolling lines. Journal of Iron and Steel Research,2019,31(02):150-158

[25] Liang S, Wang B, Chen X (2020) Design and configuration of process parameters for hot-rolled stainless steel laminar flow cooling unit. Special Steel Technology, 26(04): 56-59+48

[26] He S, Zang Y W, Zhang X R (2020) Application of water pump variable frequency speed regulation in energy saving control. Yunnan Metallurgy, 49(05):112-116

[27] Wang T, Yang J (2019) Optimal scheduling model and application of water supply pumping station in $\mathrm{M}$ city. Water Resource and Rower, 37(07): 141-145

[28] Liu Y X, Guo L, Wang C S (2018) Two-stage robust optimal economic dispatch method for microgrid. Proceedings of the CSEE, 38(14): 4013-4022+4307

[29] Wei W, Mei S, Wu L (2017) Optimal Traffic-Power Flow in Urban Electrified Transportation Networks. IEEE Transactions on Smart Grid, 8(1):84-95

[30] Pei Y, Lei Z L, Zeng Q H, Wu Y X, Lu Y L (2021) Load forecasting of refrigerated display cabinet based on CEEMDIPSO-LSTM combined model. Open Physics, 19(1):

[31] Ong K M, Ong P, Sia C K (2020) A carnivorous plant algorithm for solving global optimization problems. Applied Soft Computing, 98(April):106833 